\title{
LONG-TERM EFFECT ON HIPPOCAMPAL VOLUME (HIPPO) AFTER PERINATAL ASPHYXIA
}

M. Benders ${ }^{1}$, N. van der $\mathrm{Aa}^{1}$, M. van de Heuvel ${ }^{2}$, N. van Haren ${ }^{2}$, H. Schnack ${ }^{2}$, H. Swaab ${ }^{3}$, M. van Handel ${ }^{2}$, M. Jongmans ${ }^{4}$, L. de Vries ${ }^{5}$

${ }^{l}$ Neonatology, Wilhelmina Children's Hospital, University Medical Center, Utrecht, ${ }^{2}$ Psychiatriy, UMC Utrecht, Utrecht, ${ }^{3}$ Clinical Child and Adolescent Studies, University Leiden, Leiden, ${ }^{4}$ Psychology Social Sciences, Special Education, Univerisity Medical Center Utrecht, ${ }^{5}$ Neonatology, Wilhelmina Children's Hospital / UMC, Utrecht, The Netherlands

Background: Neonatal encephalopathy (NE) following perinatal asphyxia (PA) in term infants is an important cause of severe and more subtle neurodevelopmental disabilities. These children might experience difficulties when they have to master complex abilities at school-age. It has been described that NE has a specific, gradual effect on memory functions and behavioral problems.

Objective: To evaluate the effect of NE on HIPPO and the relation with memory tests.

Design/methods: We included 26 children with mild (NE1), 28 with moderate (NE2)

PA, and 36 controls (CTRL) (mean age: 9,9+0.05years). Children were assessed on general intellectual abilities (short-form WISC), verbal memory (digit span forward), visuo-spatial memory (short-term: Kaufman ABC; long-term: Rey Visual Design Learning Test (RVDLT) and verbal associative learning (RAKIT). A 3D-volumetric MRI was obtained.

Left and right hippocampal and total cerebral volume (TBV) were obtained (Freesurfer). Volume differences between groups were corrected for age, gender and TBV (SPSS).

Results: Significant differences were found for reduced HIPPO's in both NE groups compared to CTRL (CTRL: $4,3 \pm 0,38 ; \mathrm{NE} 1: 4,1 \pm 0.5 ; \mathrm{NE} 2: 3,8 \pm 0,6 \mathrm{ml}, p<0.001$ ). There was a significant correlation between IQ and TBV (R $0.439 p<0.001)$. HIPPO did influence RAKIT (R0,510; $<0.05)$, and mainly the right HIPPO was related to RVDLT recall and total $(\mathrm{R} 0,537 ; p<0.05)$ corrected for gender, age and TBV.

Conclusions: Hippocampal abnormalities were found between controls and NE infants after correction for gender and age. There appears to be an association between reduced HIPPOs and poorer memory functioning. 